\title{
Modelagem da viscosidade em mistura polivinilpirrolidona/quitosana para processamento de membrana adesiva
}

\author{
Viscosity modeling in polyvinylpyrrolidone/chitosan blend for adhesive membrane processing \\ Modelado de viscosidad en mezcla de polivinilpirrolidona/quitosano para procesamiento de
} membranas adhesivas

\section{Resumo}

Os biopolímeros polivinilpirrolidona e quitosana são biomateriais utilizados no tratamento de feridas, devido suas propriedades biológicas, físico-química entre outras, além de sua processabilidade. O controle da viscosidade dos polímeros é de fundamental importância para o processamento e consequentemente das propriedades das membranas, como a adesividade, crucial para aplicações de médicas em feridas. Nesse sentido, a proposta da pesquisa foi simular e avaliar a viscosidade de misturas poliméricas de quitosana de média massa molar (QM) e polivinilpirrolidona (PVP) em diferentes concentrações e proporções para síntese de membranas adesivas. Para tal, foi avaliado na literatura a viscosidade dos polímeros puros, simulado um modelo linear para viscosidade das misturas e, posteriormente, aplicado a metodologia de superfície de resposta (MSR) utilizando o modelo composto central, para três proporções QM/PVP em diferentes concentrações das soluções. A viscosidade dos polímeros QM e o PVP apresentam uma linha de tendência de crescimento exponencial, com baseado em dados da literatura, tendo um coeficiente de regressão satisfatório, e o modelo linear de simulação para viscosidade das misturas, demonstrou ser satisfatórios, considerando uma variação de $5 \%$. Os gráficos de superfície resposta e contorno deixam evidentes o efeito da concentração da solução e proporção de mistura polimérica, e em quais situações de mistura é possível atingir níveis mais elevados e reduzidos da viscosidade. As informações obtidas na pesquisa são extremamente úteis, auxiliando no desenvolvimento das misturas poliméricas, de forma, a buscar as melhores condições, reduzir tempo de experimentos focando pontos de interesse.

Palavras-chave: Quitosana; Polivinilpirrolidona; Viscosidade; Otimização; Misturas.

\footnotetext{
Abstract

Polyvinylpyrrolidone and chitosan biopolymers are biomaterials used in wound treatment, due to their biological, physicochemical, and other properties, in addition to their processability. Controlling the viscosity of polymers is of fundamental importance for processing and consequently for the properties of membranes, such as adhesiveness, crucial
} 
for medical applications in wounds. In this sense, the research proposal was to simulate and evaluate the viscosity of polymeric mixtures of medium molar mass (QM) chitosan and polyvinylpyrrolidone (PVP) in different concentrations and proportions for the synthesis of adhesive membranes. To this end, the viscosity of pure polymers was evaluated in the literature, a linear model for the viscosity of the mixtures was simulated and, subsequently, the response surface methodology (MSR) was applied using the central composite model, for three QM/PVP ratios at different concentrations of the solutions. The viscosity of QM and PVP polymers present an exponential growth trend line, based on literature data, having a satisfactory regression coefficient, and the linear simulation model for viscosity of the mixtures, proved to be satisfactory, considering a variation of 5\%. The surface response and contour graphs make evident the effect of solution concentration and polymer mix ratio, and in which mixing situations it is possible to achieve higher and lower viscosity levels. The information obtained in the research is extremely useful, aiding in the development of polymeric mixtures, in order to seek the best conditions, reduce the time of experiments focusing on points of interest.

Keywords: Chitosan; Polyvinylpyrrolidone; Viscosity; Optimization; Mixtures.

\section{Resumen}

Los biopolímeros de polivinilpirrolidona y quitosano son biomateriales utilizados en el tratamiento de heridas, debido a sus propiedades biológicas, fisicoquímicas y otras, además de su procesabilidad. El control de la viscosidad de los polímeros es de fundamental importancia para el procesamiento y, en consecuencia, para las propiedades de las membranas, como la adhesividad, crucial para aplicaciones médicas en heridas. En este sentido, la propuesta de investigación fue simular y evaluar la viscosidad de mezclas poliméricas de quitosano de masa molar media (QM) y polivinilpirrolidona (PVP) en diferentes concentraciones y proporciones para la síntesis de membranas adhesivas. Para ello, se evaluó en la literatura la viscosidad de polímeros puros, se simuló un modelo lineal para la viscosidad de las mezclas y, posteriormente, se aplicó la metodología de superficie de respuesta (MSR) utilizando el modelo compuesto central, para tres QM / PVP proporciones a diferentes concentraciones de las soluciones. La viscosidad de los polímeros QM y PVP presenta una línea de tendencia de crecimiento exponencial, con base en los datos de la literatura, teniendo un coeficiente de regresión satisfactorio, y el modelo de simulación lineal para la viscosidad de las mezclas, resultó satisfactorio, considerando una variación del 5\%. La respuesta de la superficie y los gráficos de contorno hacen evidente el efecto de la concentración de la solución y la relación de mezcla del polímero, y en qué situaciones de mezcla es posible lograr niveles de viscosidad más altos y más bajos. La información obtenida en la investigación es sumamente útil, ayudando en el desarrollo de mezclas poliméricas, con el fin de buscar las mejores condiciones, reducir el tiempo de experimentos enfocados en puntos de interés.

Palabras clave: Quitosano; Polivinilpirrolidona; Viscosidad; Mejoramiento; Mezclas.

\section{Introdução}

Os biomateriais podem ser definidos como qualquer material natural ou sintético que interagem com sistemas biológicos por um período significativo, visando tratar, aumentar ou substituir um órgão, tecido ou função do corpo (Pascual-Gil et al., 2015; Williams, 2009). Esses quando implantados em partes danificadas do corpo e devem ser biocompatíveis com os tecidos do corpo, ou seja, com o mínimo de reações alérgicas, inflamatórias ou tóxicas e biofuncionais desempenhando apropriadamente a função, dada as suas propriedades mecânicas, físicas e químicas (Agência Brasileira de Desenvolvimento Industrial, 2011; Raut et al., 2020; Rehman et al., 2018; Zeugolis et al., 2015).

Dentre a classificação dos biomateriais, os polímeros são os mais empregados no âmbito médico, a sua ampla variabilidade de suas propriedades mecânicas, químicas, físicas, biológicas e reológicas, facilidade de processamento para produzir formas variadas (partículas, filmes, fios, membranas, hidrogéis dentre outros), possuir origem natural, sintética e artificial ou natural modificado, além da possibilidade de formação de blendas e compósitos, para otimizar propriedades (Bernardo et al., 2021; Brito et al., 2011; Callister Jr et al., 2020; Oréfice et al., 2006; Pires et al., 2015).

Um campo de interesse para aplicação dos biomateriais poliméricos é no desenvolvimento de curativos com ênfase para o tratamento de feridas e lesões de pele, com o propósito de prevenir a infecção e complicações promovendo a cicatrização, além de apresentarem atividades biológicas como fungicida e bactericida, neste sentido, esses biomateriais destacam-se, por ser produzidos em forma de membranas, hidrogéis, filmes, entre outras e associados a substâncias como íons de prata para melhorarem propriedades em busca de benefícios para o tratamento de feridas (Araújo et al., 2017; Augusto et al., 2021; Pinheiro et al., 2018; Tang et al., 2010).

Nesse âmbito, os polímeros quitosana e o polivinilpirrolidona que apresentam propriedades favoráveis ao 
desenvolvimento de curativos e sendo averiguadas em outras áreas da engenharia de tecidos. O polivinilpirrolidona (PVP) é um homopolímero de N-vinil-2-pirrolidona, polímero amorfo, sintético da família dos vinílicos, obtido através da polimerização radicalar em água ou álcool isopropílico, compatível com a maioria das resinas e solventes devido a seu componente hidrofílico (pirrolidona) e hidrofóbico (grupo alquil). Possui propriedades inerentes como formação de filme fino, adesividade, biocompatibilidade, não toxidade, estabilidade em diversos $\mathrm{pH}$, e resistência mecânica demonstrando ser um biomaterial eficiente (Franco et al., 2020; Kurakula et al., 2020; Mishra et al., 2019; Sizílio et al., 2018).

Por sua vez, a quitosana é um biopolímero de origem natural derivado do tratamento de desacetilação alcalina parcial ou total da quitina, onde as ligações $\mathrm{N}$-acetil da quitina são rompidas gerando D-glicosamina contendo um grupo amino livre, a qual é o segundo polissacarídeo mais abundante, apenas atrás da celulose. Apresenta comportamento catiônico, semicristalino, além de manter propriedades intrínsecas para aplicações biomédicas como: biocompatibilidade, biodegradabilidade, atoxidade e atividade antimicrobiana, tendo suas propriedades físico-químicas, biológicas e de processabilidade influenciadas pela massa molar média e grau médio de acetilação (Dallan, 2005; Damian et al., 2005; Franco et al., 2020; Muxika et al., 2017; Spin-Neto et al., 2008; Tomaz, 2017).

Os biomateriais de quitosana estão presentes na forma de membranas porosas, não-tecidos, nanopartículas, nanofibras, e filmes são usados em medicina regenerativa, engenharia de tecidos, liberação controlada de fármacos e em biossensores, em razão dessas características, é avaliado a mistura de polímeros sintéticos e naturais para otimizar o processo de cicatrização da ferida ou lesão cutânea (Bernardo et al., 2021).

A adesão é um fenômeno como objetivo de unir componentes, sendo de grande interesse em todas as áreas do conhecimento, inclusive a biomédica, que contribui para conferir resistência, biomateriais com adesividade apropriadas para a aplicação desejada, substituindo técnicas tradicionais de fixação mecânica, melhorando o processo de cicatrização e diminuição nas trocas. Curativos com excelente aderência à base da lesão de pele ou em determinados tipos de feridas possibilita a não contaminação externa e perda de líquidos, que favorecem o processo de cicatrização e promove a redução da necessidade de substituição dos curativos (Bharti, 2018; Pereira et al., 2019).

Dessa maneira, o presente trabalho realizar a modelagem na viscosidade de misturas de quitosana e polivinilpirrolidona (PVP) e aplicação da com a metodologia de superfície resposta utilizando o modelo de composto central, avaliando a influência dos fatores concentração da solução e proporção na mistura polimérica para avaliar as condições mais adequadas para o processamento de membranas adesivas com possíveis aplicações em curativos em feridas.

\section{Metodologia}

Para atender os objetivos propostos, foram determinadas as propriedades de viscosidade da quitosana de médio peso molar (QM) em solução de ácido acético à (1\%) e do polivinilpirrolidona (PVP) K - 90 em solução aquosa, ambos a $25^{\circ} \mathrm{C}$, encontrados na literatura e em especificações técnicas divulgados por empresas, para 5 concentrações diferentes, com base nessas informações foram construídas as curvas de viscosidade das soluções poliméricas puras, determinando as equações de regressão e determinação.

Posteriormente, baseado na viscosimetria de soluções diluídas a determinação da viscosidade das misturas poliméricas pode ser definida pela equação clássica de Huggins Equação 1 (Penha et al., 2020; Santos et al., 2003).

$$
\boldsymbol{\eta}_{s p}=[\boldsymbol{\eta}] \boldsymbol{C}+\boldsymbol{k}[\boldsymbol{\eta}] \boldsymbol{C}^{2}
$$

onde $n_{s p}$ é a viscosidade específica, [ $\eta$ ] é a viscosidade intrínseca e C é a concentração, enquanto que o valor de k é o coeficiente angular de Huggins. 
As determinações das viscosidades para as misturas poliméricas foram realizadas assumindo que, a mesma possui um comportamento linear em relação à viscosidade intrínseca e a composição das soluções poliméricas puras, ou seja, no caso em que a Equação (2) fosse válida:

$$
[\eta]=\mathbf{X}_{\mathrm{PVP}}[\eta]_{\mathrm{PVP}}+\mathbf{X}_{\text {quit }}[\boldsymbol{\eta}]_{\text {quit }}
$$

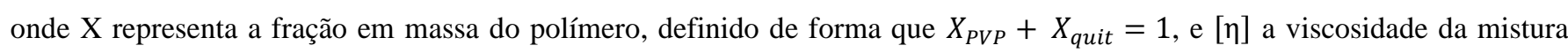
polimérica.

Em seguida foi aplicado a metodologia de superfície resposta (MSR) utilizando o modelo composto central (Tabela 1), determinando as viscosidades das misturas poliméricas proporções de solução polimérica, denominada: mistura $\mathrm{A}(90 \% \mathrm{Q}+$ $10 \% \mathrm{P})$, mistura $\mathrm{B}(50 \% \mathrm{Q}+50 \% \mathrm{P})$ e mistura $\mathrm{C}(10 \% \mathrm{Q}+90 \% \mathrm{P})$, onde $(\mathrm{Q})$ é a quitosana e $(\mathrm{P})$ é o polivinilpirrolidona nas diferentes concentrações.

Tabela 1 - Modelo composto Central para a MSR.

\begin{tabular}{ccc}
\hline Fatores & Concentração & Nível \\
\hline \multirow{2}{*}{$\begin{array}{ccc}\text { Polivinilpirrolidona: } \\
\text { CP }\end{array}$} & 5,00 & -1 (baixo) \\
\cline { 2 - 3 } & 10,00 & 0 (médio) \\
\hline Quitosana: & 15,00 & +1 (alto) \\
\cline { 2 - 3 } CQ & 0,5 & -1 (baixo) \\
\cline { 2 - 3 } & 1,0 & 0 (médio) \\
\hline
\end{tabular}

Fonte: Autores.

\section{Resultados e Discussão}

A Figura 1, ilustra o perfil da viscosidade da quitosana média massa molar em solução de ácido acético à (1\%) e $25^{\circ} \mathrm{C}$, de acordo com dados encontrados na literatura e de especificações técnicas para 5 concentrações $\mathrm{m} / \mathrm{V}$ (Hu et al., 2007; Pillai et al., 2009; Sharma et al., 2011; Sigma-Aldrich, 2020).

Figura 1 - O gráfico do perfil de viscosidade da quitosana.

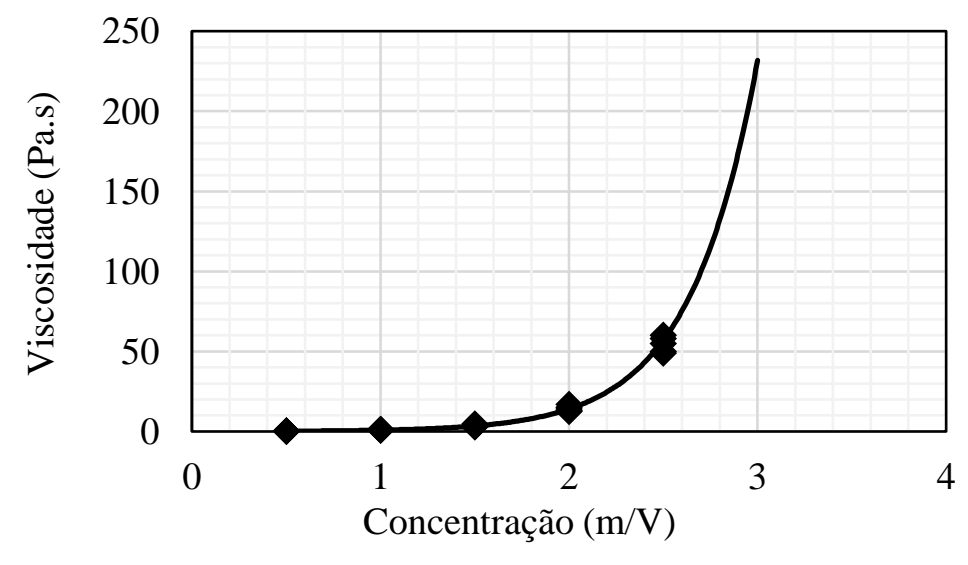

Fonte: Autores. 
Foi determinado a linha de tendência (Figura 1) que melhor representam-se as viscosidades nas respectivas concentrações observadas, sendo o modelo exponencial o que apresentou o melhor resultado e então determinado a Equação 3 e o coeficiente de determinação $\left(\mathrm{R}^{2}\right)$ que foi igual da 0,93 .

$$
[\eta]_{\text {quit }}=0,019 * e^{-3,205 \text { Cquit }}
$$

Spin-Neto et. al., $2008 \mathrm{em}$ seu estudo sobre biomateriais a base de quitosana, abordou que fatores como: $\mathrm{pH}$ do solvente utilizado, concentração e massa molecular estão diretamente relacionados à viscosidade do gel obtido, bem como às propriedades do biomaterial final, corroborando com o efeito observado da elevação da viscosidade com o aumento da concentração da quitosana na solução.

A Figura 2, ilustra o perfil da viscosidade do polivinilpirrolidona PVP K-90 em solução de ácido aquosa à $\left(25^{\circ} \mathrm{C}\right)$, de acordo com dados encontrados na literatura e de especificações técnicas para 5 concentrações m/V (Ashland, 2020).

Figura 2 - O gráfico do perfil de viscosidade do PVP K-90.

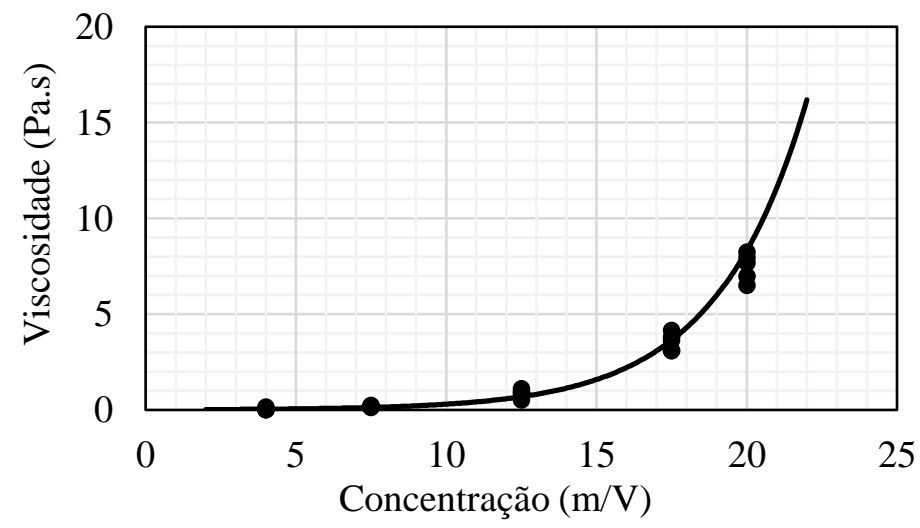

Fonte: Autores.

Foi determinado a linha de tendência (Figura 2) que melhor representam-se as viscosidades nas respectivas concentrações observadas, sendo o modelo exponencial o que apresentou o melhor resultado e então determinado a Equação $4 \mathrm{e}$ o coeficiente de determinação $\left(\mathrm{R}^{2}\right)$ que foi igual da 0,98 .

$$
[\eta]_{p v p}=0,011 * e^{-0,332 * C p v p}
$$

Higashi et al., 2020 mostrou que à medida que a concentração PVP diminui em uma solução a uma redução da viscosidade, corroborando com o resultado observado.

Os resultados para viscosidade das misturas polimérica considerando uma variação de $\pm 5 \%$ segundo a equação (2) associadas as equações 3 e 4, são apresentados na Tabela 2, onde o modelo de composto central para análise da metodologia de superfície resposta é aplicada para as diferentes proporções de solução na mistura, para os fatores (CQ e CP) em níveis (baixo, médio e alto de concentrações). 
Tabela 2 - Viscosidade das misturas poliméricas A, B e C aplicadas no modelo composto central em triplicata.

\begin{tabular}{|c|c|c|c|c|c|c|c|c|c|c|}
\hline \multicolumn{2}{|c|}{ Fatores e Níveis } & \multicolumn{9}{|c|}{ Viscosidade (Pa.S) } \\
\hline $\mathrm{CQ}$ & $\mathbf{C P}$ & \multicolumn{3}{|c|}{ Mistura A } & \multicolumn{3}{|c|}{ Mistura B } & \multicolumn{3}{|c|}{ Mistura C } \\
\hline 0 & 0 & 0,46 & 0,44 & 0,48 & 0,39 & 0,37 & 0,41 & 0,32 & 0,30 & 0,34 \\
\hline-1 & -1 & 0,09 & 0,09 & 0,09 & 0,08 & 0,08 & 0,08 & 0,06 & 0,06 & 0,06 \\
\hline 0 & 0 & 0,44 & 0,42 & 0,46 & 0,38 & 0,35 & 0,39 & 0,31 & 0,29 & 0,32 \\
\hline 1 & 1 & 2,29 & 2,18 & 2,40 & 1,97 & 2,07 & 1,87 & 1,66 & 1,74 & 1,58 \\
\hline 0 & -1 & 0,43 & 0,41 & 0,45 & 0,27 & 0,26 & 0,28 & 0,10 & 0,10 & 0,11 \\
\hline 1 & 0 & 2,16 & 2,05 & 2,27 & 1,33 & 1,26 & 1,40 & 0,51 & 0,48 & 0,54 \\
\hline-1 & 1 & 0,24 & 0,23 & 0,25 & 0,84 & 0,80 & 0,88 & 1,43 & 1,36 & 1,50 \\
\hline 1 & -1 & 0,29 & 0,28 & 0,30 & 1,21 & 1,15 & 1,27 & 2,13 & 2,02 & 2,24 \\
\hline 0 & 1 & 0,59 & 0,56 & 0,62 & 1,03 & 0,98 & 1,08 & 1,47 & 1,40 & 1,54 \\
\hline 0 & 0 & 0,44 & 0,42 & 0,46 & 0,37 & 0,35 & 0,39 & 0,30 & 0,29 & 0,32 \\
\hline 0 & 0 & 0,44 & 0,46 & 0,51 & 0,37 & 0,39 & 0,43 & 0,30 & 0,32 & 0,35 \\
\hline 0 & 0 & 0,48 & 0,46 & 0,51 & 0,41 & 0,39 & 0,43 & 0,33 & 0,32 & 0,35 \\
\hline-1 & 0 & 0,12 & 0,11 & 0,13 & 0,20 & 0,19 & 0,21 & 0,28 & 0,27 & 0,29 \\
\hline
\end{tabular}

Fonte: Autores.

As Equações de regressão (5, 6 e 7) foram obtidas pela MSR aplicando o método de composto central para as misturas poliméricas $\mathrm{A}, \mathrm{B}$ e $\mathrm{C}$ respectivamente.

$$
\begin{aligned}
{[\eta]_{A}=} & 0,52+0,72 C Q+0,38 C P+0,46 C Q^{2}-0,17 C P^{2}+0,46 C Q C P \\
& {[\eta]_{B}=0,39+0,56 C Q+0,38 C P+0,38 C Q^{2}+0,26 C P^{2} } \\
{[\eta]_{C}=} & 0,25+0,42 C Q+0,38 C P+0,30 C Q^{2}+0,69 C P^{2}-0,46 C Q C P
\end{aligned}
$$

Os coeficientes de determinação $\left(\mathrm{R}^{2}\right)$ para as misturas poliméricas $\mathrm{A}, \mathrm{B}$ e C foram $0,88,0,99$ e 0,88 respectivamente, os resultados mostram que para o modelo linear de viscosidade (Equação 2), os resultados indicam que todas as misturas poliméricas são explicadas satisfatoriamente, conduto, a mistura onde a fração de massa do polímero é semelhante, o modelo possui resultado superior.

Na Figura 3, observam-se os gráficos de Pareto que determinar a magnitude e a importância dos efeitos. Para as misturas poliméricas $\mathrm{A}, \mathrm{B}$ e $\mathrm{C}$, verifica-se que apenas o fator $\mathrm{CP}^{2}$ para a mistura $\mathrm{A}$ e B não possui significância ao nível $\alpha$ de 0,05 , pois seu valor é inferior ao limite de 2,04 (linha de referência), os demais fatores são significativos ao nível 0,05. 
Figura 3 - Gráficos de Pareto para as misturas poliméricas A, B e C respectivamente,
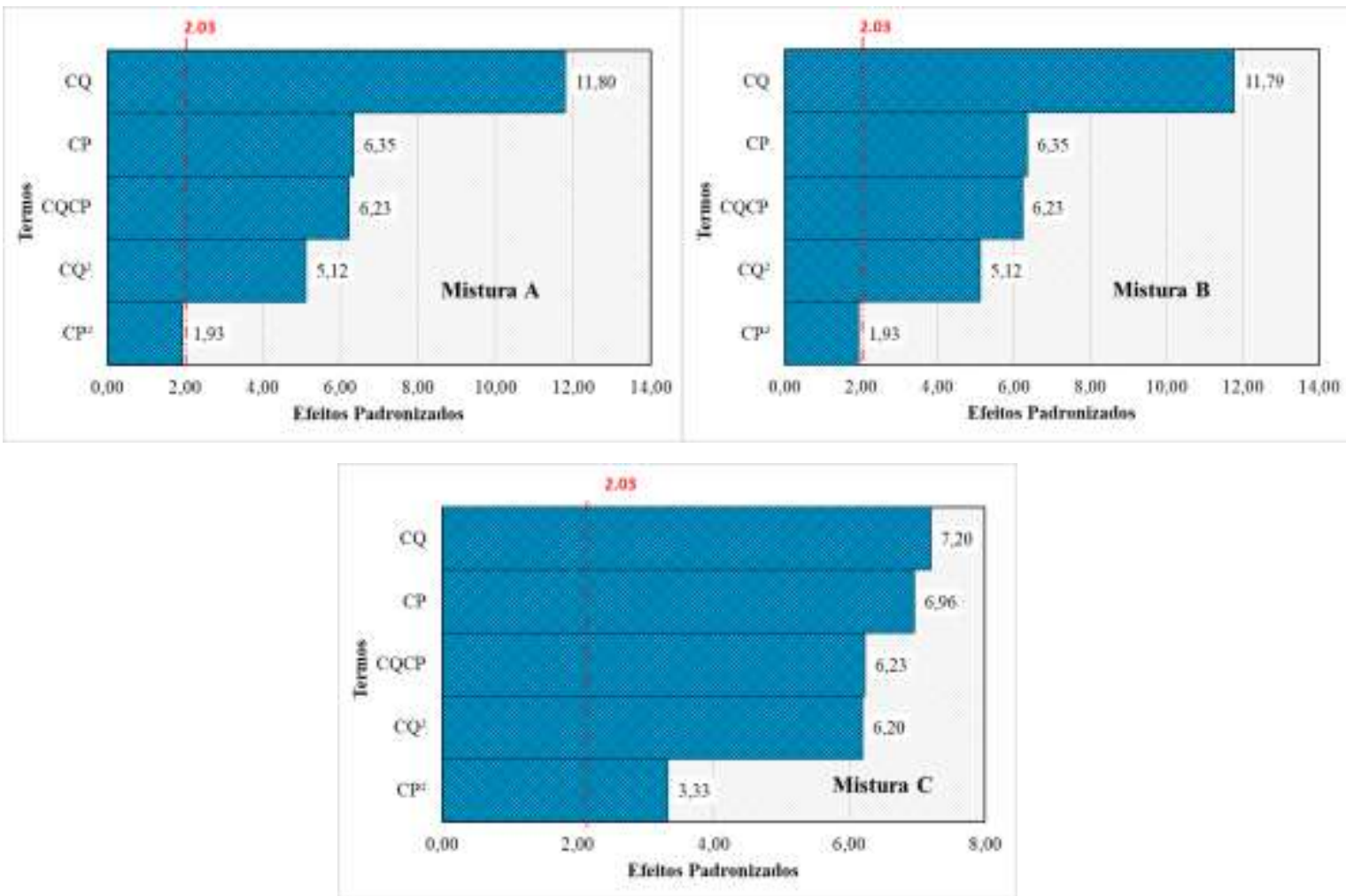

Fonte: Autores.

Os resultados da análise de variância (ANOVA) para as simulações das misturas poliméricas A, B e C são apresentados na Tabela 3, observa-se que para o grau de liberdade 1 e 33 do erro com 95\% de confiança o valor da distribuição estatística tabelado é de 4,12, logo a ANOVA indica que apenas o fator quadrado $\mathrm{CP}^{2}$ da mistura $\mathrm{A}$ e $\mathrm{B}$ possuem valor $\mathrm{F}$ estatísticos inferior ao estatístico tabelado, sendo este fator não significativo, corroborando com o resultado apresentado no gráfico de Pareto (Figura $3)$. 
Tabela 3 - Análise de variância para simulação da mistura polimérica A, B e C.

\begin{tabular}{cccccc}
\hline \multicolumn{5}{c}{ Mistura A } \\
\hline Fatores & Grau de Liberdade & Soma dos Quadrados & Média dos Quadrados & Valor F & Valor P \\
\hline $\mathrm{CQ}$ & 1 & 9,20 & 9,20 & 139,19 & 0,00 \\
\hline $\mathrm{CP}$ & 1 & 2,67 & 2,67 & 40,36 & 0,00 \\
\hline $\mathrm{CQ}^{2}$ & 1 & 1,73 & 1,73 & 26,22 & 0,00 \\
\hline $\mathrm{CP}^{2}$ & 1 & 0,25 & 0,25 & 3,73 & 0,06 \\
\hline $\mathrm{CQCP}$ & 1 & 2,57 & 2,57 & 38,83 & 0,00 \\
\hline Erro & 33 & 2,18 & 0,07 & \\
\hline Total & 38 & 18,35 & & \\
\hline
\end{tabular}

Mistura B

\begin{tabular}{cccccc}
\hline Fatores & Grau de Liberdade & Soma dos Quadrados & Média dos Quadrados & Valor F & Valor P \\
\hline $\mathrm{CQ}$ & 1 & 11,87 & 5,94 & 89,77 & 0,00 \\
\hline $\mathrm{CP}$ & 1 & 9,20 & 9,20 & 139,19 & 0,00 \\
\hline $\mathrm{CQ}^{2}$ & 1 & 2,67 & 2,67 & 40,36 & 0,00 \\
\hline $\mathrm{CP}^{2}$ & 1 & 1,73 & 1,73 & 26,22 & 0,00 \\
\hline $\mathrm{CQCP}$ & 1 & 0,25 & 0,25 & 3,73 & 0,06 \\
\hline Erro & 33 & 2,57 & 2,57 & 38,83 & 0,00 \\
\hline Total & 38 & 2,18 & 0,07 & &
\end{tabular}

Mistura C

\begin{tabular}{cccccc}
\hline Fatores & Grau de Liberdade & Soma dos Quadrados & Média dos Quadrados & Valor F & Valor P \\
\hline $\mathrm{CQ}$ & 1 & 3,20 & 3,20 & 48,44 & 0,00 \\
\hline $\mathrm{CP}$ & 1 & 2,57 & 2,57 & 38,88 & 0,00 \\
\hline $\mathrm{CQ}^{2}$ & 1 & 0,73 & 0,73 & 11,11 & 0,00 \\
\hline $\mathrm{CP}^{2}$ & 1 & 3,94 & 3,94 & 59,59 & 0,00 \\
\hline $\mathrm{CQCP}$ & 1 & 2,54 & 2,54 & 38,43 & 0,00 \\
\hline Erro & 33 & 2,18 & 0,07 & \\
\hline Total & 38 & 17,47 & & & \\
\hline
\end{tabular}

Fonte: Autores.

Na Figura 4, são ilustrados os gráficos de contorno e de superfície resposta obtidos na MSR pelo modelo composto central para as misturas poliméricas A, B e C, onde se verifica que os níveis altos para CP e CQ promovem a uma elevação da viscosidade, aumento da proporção da solução de PVP tende a reduzir a viscosidade e o aumento da proporção da solução de quitosana de média massa molar a aumentar a viscosidade. Esses resultados corroboram com Spin-Neto 2008, onde se observa que a concentração polimérica de uma solução, tende a elevar a viscosidade, e com Higashi et. al. 2020, em que observou o efeito da concentração do PVP na redução da viscosidade. 
Figura 4 - Os gráficos de contorno e superfície resposta para as misturas poliméricas A, B e C respectivamente.
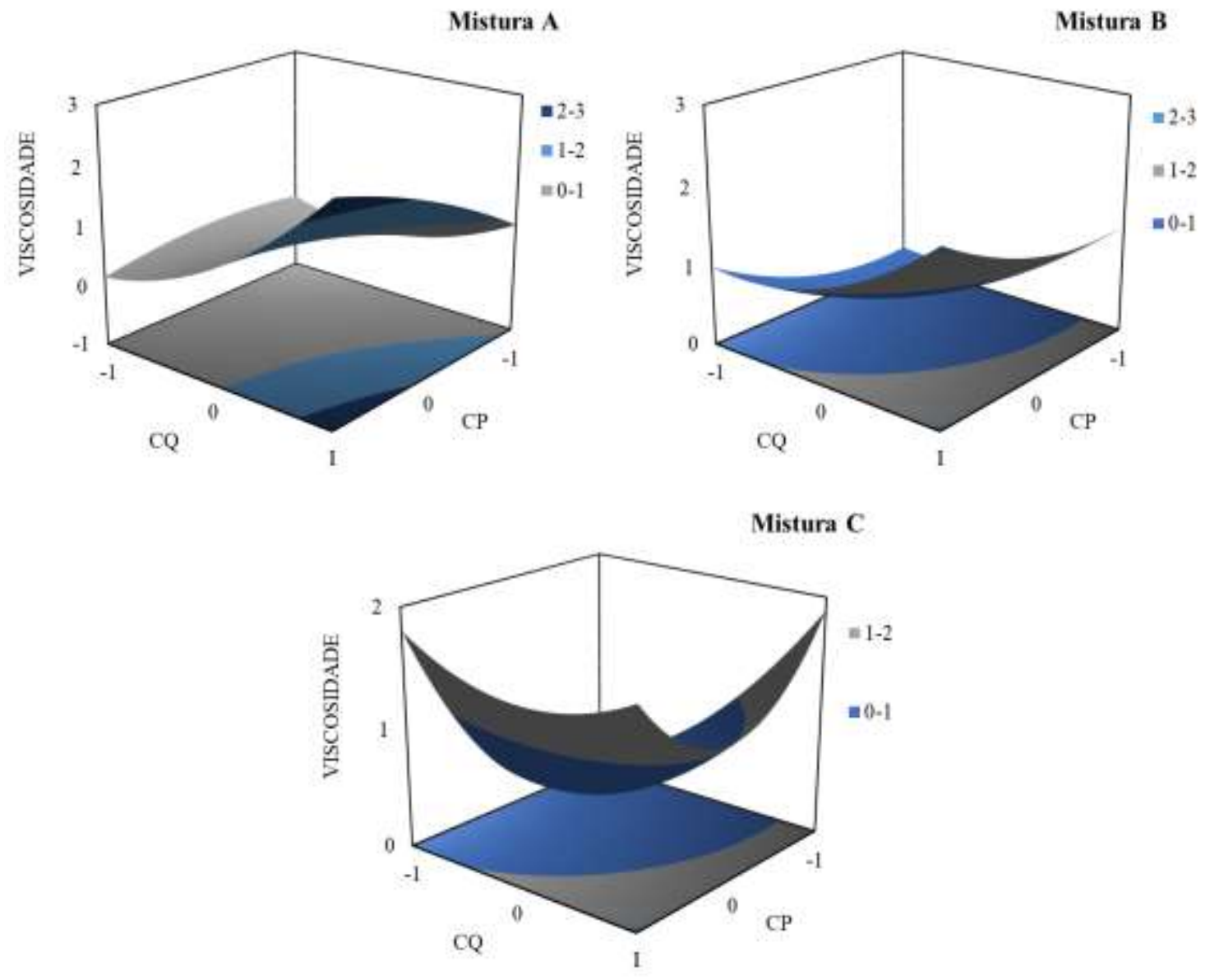

Fonte: Autores.

Para mistura C, observa-se um efeito, parábola, ao ter, um nível alto para o fator CP e variamos os níveis do fator CQ ou vice-versa, esse efeito deve-se provavelmente pela viscosidade próxima entre os polímeros com concentrações diferentes, ou seja, PVP K - 90 com concentração m/V entre 5 - $15 \%$ possui uma viscosidade próxima à da quitosana com concentração m/V entre $0,5-1,5 \%$ e a proporção de solução na mistura que contém 90\% de PVP K - 90.

A viscosidade das soluções é uma das variáveis que caracteriza reologicamente uma substância, ou seja, a propriedade reológica, amplamente aplicada no processamento dos polímeros e podem promover modificações físicas e químicas nos produtos formados. Dessa forma, a viscosidade pode ser utilizada para controlar a coesão e aderência dos materiais poliméricos e consequentemente as propriedades adesivas. Estudos sobre reologia dos polímeros mostram que concentração, massa molar, densidade, tipo de solvente, complexidades das cadeias poliméricas afetam na viscosidade dos polímeros (Damian et al., 2005).

Os resultados são promissores para controle de processamento, Sharma et al., 2011, menciona a importância de modelar a viscosidade do polímero com precisão para que previsões precisas da taxa de injeção possam ser feitas, ou seja, para representação do processamento, como a distribuição de pressão substancialmente longe do poço, influenciando significativamente o desempenho de processos químicos.

\section{Conclusão}

A viscosidade dos polímeros quitosana de média massa molar e o polivinilpirrolidona PVP K - 90 apresentam uma linha de tendência de crescimento exponencial, com baseado em dados da literatura, tendo um coeficiente de regressão satisfatório. 
A partir da metodologia de superfície resposta utilizando o modelo de composto central para avaliar o efeito do fator concentração da solução de quitosana e PVP - K 90 na viscosidade para as diferentes proporções poliméricas. Os resultados de superfície resposta e contorno deixam evidentes o efeito da concentração da solução polimérica e da proporção de mistura, e em quais situações de mistura é possível atingir níveis mais elevados e reduzidos na viscosidade. As informações obtidas na pesquisa são extremamente úteis, auxiliando no desenvolvimento de formulações de misturas poliméricas com viscosidade adequada para o processamento e controle de propriedades, otimizando os experimentos.

Trabalhos futuros com estudos experimentais da viscosidade para comprovação da modelagem proposta baseada em dados teóricos, assim como caracterizações das físico químicas e mecânicas de membranas obtidas das misturas poliméricas são relevantes e promissores.

\section{Referências}

Agência Brasileira de Desenvolvimento Industrial. (2011). Manual de Registro e Cadastramento de Materiais de Uso em Saúde (ABDI).

Araújo, Â. M., Letícia, I., Palmeira, T., \& Maria, A. (2017). Utilização de nanopartículas no tratamento de feridas : revisão sistemática. 1-10.

Ashland. (2020). PVP Polyvinylpyrrolidone - Polymers Intermediates, solvents, monomers, polymers and specialty. Ashland Brochure. https://www.brenntag.com/media/documents/bsi/product_data_sheets/material_science/ashland_polymers/pvp_polymers_brochure.pdf

Augusto, F., \& Leite, S. (2021). Curativos de prata no tratamento de feridas exsudativas - uma revisão sistemática. Revista Feridas, 09(46), 1682-1689.

Bernardo, M., Paschoalin, R., Santos, D., Bilatto, S., Farinas, C., Correa, D., Oliveira, O., \& Mattoso, L. (2021). Processamento e Aplicação de Biomateriais Poliméricos: Avanços Recentes e Perspectivas. Química Nova, January. 10.21577/0100-4042.20170781

Bharti, S. (2018). Adhesives and Adhesion Technologies: A Critical Review. American Journal of Polymer Science and Technology, 4(1), 36. 10.11648/j.ajpst.20180401.13

Brito, G. F., Agrawal, P., Araújo, E. M., \& Mélo, T. J. A. (2011). Biopolímeros , Polímeros Biodegradáveis e Polímeros Verdes. Revista Eletrônica de Materiais e Processos, 2, 127-139. 1809-8797

Callister Jr, W. D., \& Rethwisch, D. G. (2020). Fundamentals of materials science and engineering: an integrated approach. John Wiley \& Sons.

Dallan, P. R. M. (2005). Síntese e caracterizaçao de membranas de quitosana para aplicaçao na regeneração da pele. Universidade Estadual de Campinas.

Damian, C., Beirão, L. H., Francisco, A. De, Santo, M. L. P. E., \& Teixeira, E. (2005). Quitosana : Um Amino Polissacarídio Com Características Funcionais. Alim. Nutr., 16(2), 195-205.

Franco, P., \& De Marco, I. (2020). The use of poly(N-vinyl pyrrolidone) in the delivery of drugs: A review. Polymers, 12(5), 18-21. 10.3390/POLYM12051114

Higashi, S., Hirai, T., Matsubara, M., Yoshida, H., \& Beniya, A. (2020). Dynamic viscosity recovery of electrospinning solution for stabilizing elongated ultrafine polymer nanofiber by TEMPO-CNF. Scientific Reports, 10(1), 1-8. 10.1038/s41598-020-69136-2

Hu, X., Du, Y., Tang, Y., Wang, Q., Feng, T., Yang, J., \& Kennedy, J. F. (2007). Solubility and property of chitin in NaOH/urea aqueous solution. Carbohydrate Polymers, 70(4), 451-458. 10.1016/j.carbpol.2007.05.002

Kurakula, M., \& Rao, G. S. N. K. (2020). Pharmaceutical assessment of polyvinylpyrrolidone (PVP): As excipient from conventional to controlled delivery systems with a spotlight on COVID-19 inhibition. Journal of Drug Delivery Science and Technology, 60(September), 102046. 10.1016/j.jddst.2020.102046

Mishra, R., Varshney, R., Das, N., Sircar, D., \& Roy, P. (2019). Synthesis and characterization of gelatin-PVP polymer composite scaffold for potential application in bone tissue engineering. European Polymer Journal, 119(July), 155-168. 10.1016/j.eurpolymj.2019.07.007

Muxika, A., Etxabide, A., Uranga, J., Guerrero, P., \& de la Caba, K. (2017). Chitosan as a bioactive polymer: Processing, properties and applications. International Journal of Biological Macromolecules, 105, 1358-1368. 10.1016/j.ijbiomac.2017.07.087

Oréfice, R. L., Pereira, M. de M., \& Mansur, H. S. (2006). Biomateriais: fundamentos e aplicações. In Biomateriais: fundamentos e aplicações (p. 538).

Pascual-Gil, S., Garbayo, E., Díaz-Herráez, P., Prosper, F., \& Blanco-Prieto, M. J. (2015). Heart regeneration after myocardial infarction using synthetic biomaterials. Journal of Controlled Release, 203, 23-38. 10.1016/j.jconrel.2015.02.009

Penha, V. C. S., Silva, Y. M. de O., Silva, G. C. da, \& Queiroz, M. B. de. (2020). Produção De Microesferas De Quitosana Para Fins Farmacêuticos. Brazilian Journal of Development, 6(8), 55941-55973. 10.34117/bjdv6n8-129

Pereira, C. C. C., \& Hentschke, G. S. (2019). O Uso da Pele de Tilápia como Curativo Biológico na Recuperação de Pacientes Queimados. Revista Das Semanas Acadêmicas, 5(2).

Pillai, C. K. S., Paul, W., \& Sharma, C. P. (2009). Chitin and chitosan polymers: Chemistry, solubility and fiber formation. Progress in Polymer Science (Oxford), 34, 641-678. 10.1016/j.progpolymsci.2009.04.001 
Pinheiro, C. J. G., Carreira, L. G., Brito, G. A. O., Baranano, A. G., Morais, P. A. B., \& Pinheiro, I. R. (2018). Preparação e caracterização de hidrogel com nanopartícula de cério, calendula officinalis e bixa orellana L. como potenciais curativos de feridas crônicas. Universidade Federal do Espírito Santo.

Pires, A. L. R., Bierhalz, A. C. K., \& Moraes, Â. M. (2015). Biomaterials: Types, Applications, and Market. Química Nova, 38(7), 957-971. 10.5935/01004042.20150094

Raut, H. K., Das, R., Liu, Z., Liu, X., \& Ramakrishna, S. (2020). Biocompatibility of Biomaterials for Tissue Regeneration or Replacement. Biotechnology Journal, 15(12), 1-14. 10.1002/biot.202000160

Rehman, M., Madni, A., \& Webster, T. J. (2018). The era of biofunctional biomaterials in orthopedics: what does the future hold? Expert Review of Medical Devices, 15(3), 193-204. 10.1080/17434440.2018.1430569

Santos, J. E. dos, Soares, J. da P., Dockal, E. R., Campana Filho, S. P., \& Cavalheiro, É. T. G. (2003). Caracterização de quitosanas comerciais de diferentes origens. Polimeros, 13(4), 242-249. 10.1590/s0104-14282003000400009

Sharma, A., Delshad, M., Huh, C., \& Pope, G. A. (2011). A practical method to calculate polymer viscosity accurately in numerical reservoir simulators. Proceedings - SPE Annual Technical Conference and Exhibition, 5, 3881-3894. 10.2118/147239-ms

Sigma-Aldrich. (2020). Product Product Specification Chitosan medium molecular weight. In Product Specification Chitosan medium molecular weight (p. 1). 10.1007/1-4020-4350-3_7

Sizílio, R. H., Galvão, J. G., Trindade, G. G. G., Pina, L. T. S., Andrade, L. N., \& Gonsalves, J. K. M. C. (2018). Chitosan / pvp-based mucoadhesive membranes as a promising delivery system of betamethasone-17-valerate for aphthous stomatitis. Carbohydrate Polymers, 190(February), 339-345. 10.1016/j.carbpol.2018.02.079

Spin-Neto, R., Pavone, C., Moreno De Freitas, R., Adriana, R., Marcantonio, C., \& Marcantonio-Júnior, E. (2008). Biomateriais à base de quitosana com aplicação médica e odontológica: revisão de literatura. Revista de Odontologia Da UNESP, 37(2), 155-161.

Tang, H., Zhang, P., Kieft, T. L., Ryan, S. J., Baker, S. M., Wiesmann, W. P., \& Rogelj, S. (2010). Antibacterial action of a novel functionalized chitosanarginine against Gram-negative bacteria. Acta Biomaterialia, 6(7), 2562-2571. 10.1016/j.actbio.2010.01.002

Tomaz, A. F. (2017). Desenvolvimento de membrana de quitosana/1, 4 naftoquinona para liberação controlada: curativo para feridas oncológicas.

Williams, D. F. (2009). On the nature of biomaterials. Biomaterials, 30(30), 5897-5909. 10.1016/j.biomaterials.2009.07.027

Zeugolis, D. I., \& Pandit, A. (2015). Biofunctional biomaterials - The next frontier. Bioconjugate Chemistry, 26(7), 1157. 10.1021/acs.bioconjchem.5b00342 